\title{
Gait deviations of patients with ruptured anterior cruciate ligament: a cross-sectional gait analysis study on male patients
}

\author{
Jay Hoon Park ${ }^{1,2}$, Min-Ho Choi ${ }^{1,2}$, Joonhee Lee ${ }^{1}$, Hyuk-Soo Han ${ }^{1,2}$, Myung Chul Lee ${ }^{1,2}$ and Du Hyun Ro ${ }^{1,2^{*}}$ (D)
}

\section{Background}

Anterior cruciate ligament (ACL) is the primary restraint of anteroposterior instability and the secondary restraint of rotational instability. Rupture of ACL results in knee instability $[1,2]$, and the instability can affect kinematics and kinetics of the knee joint [3-12]. Kinematics describe the different angulation of the knee joint, whereas kinetics explain the change in knee joint moment.

Gait deviation in patients with ACL rupture was first reported by Berchuck et al. in 1990 [6]. They proposed a "quadriceps avoidance gait," wherein patients with ACL rupture walked with reduced quadriceps activation to decrease anterior tibial translation. It is the most-cited gait adaptation mechanism of ACL rupture patients. However, subsequent research raised counterarguments $[5-11,13]$. Firstly, several researchers noted the absence the of quadriceps avoidance gait pattern in their group of patients $[7,9,12,13]$. Secondly, knee was extended at the initial stance phase, as opposed to the mid-stance phase reported by Berchuck $[8,10,14,15]$. It is worthwhile noting that the methodology among studies is inconsistent. Patient selection criteria, gait examination timing, and diagnostic method varied among the studies, and conclusions were inconsistent. Currently, the presence of quadriceps avoidance gait as well as the gait adaptation

*Correspondence: duhyunro@gmail.com

${ }^{1}$ Department of Orthopaedic Surgery, Seoul National University Hospital, 101 Daehak-ro, Jongno-gu, Seoul 110-744, Korea

Full list of author information is available at the end of the article mechanisms after ACL rupture is inconsistent across the literature [3-13].

Information regarding the change in gait pattern may provide further insight into neuromuscular strategies of patients with ACL rupture, in addition to shedding light on the pathophysiology, by which arthritis tends to develop in ACL-deficient knees. Therefore, in the present study, we performed a comprehensive analysis of knee biomechanics using principal component analysis (PCA). Unlike simple comparison of maximal or minimal values, PCA can analyze a large matrix of data without loss of information.

In this study, we tested the hypothesis that kinematic and kinetic deviations of ACL rupture exist, and that these deviations may act as a stabilization strategy. The purpose of this study was to find kinematic and kinetic deviations of ACL-ruptured knees by comparing them with their contralateral uninjured knee in the axial and sagittal planes.

\section{Methods}

Participants

This cross-sectional gait analysis study was conducted on 114 patients with complete unilateral ACL rupture (Fig. 1). ACL rupture was diagnosed clinically and confirmed by magnetic resonance imaging (MRI). MRI criteria applied to assess ACL rupture were fiber discontinuity, abnormal orientation of ACL with respect the Blumensaat's line, and empty notch sign. To minimize possible confounding effects $[3,4,16]$, patients

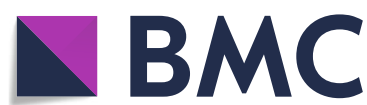

Part of Springer Nature

(c) The Author(s) 2021. Open Access This article is licensed under a Creative Commons Attribution 4.0 International License, which permits use, sharing, adaptation, distribution and reproduction in any medium or format, as long as you give appropriate credit to the original author(s) and the source, provide a link to the Creative Commons licence, and indicate if changes were made. The images or other third party material in this article are included in the article's Creative Commons licence, unless indicated otherwise in a credit line to the material. If material is not included in the article's Creative Commons licence and your intended use is not permitted by statutory regulation or exceeds the permitted use, you will need to obtain permission directly from the copyright holder. To view a copy of this licence, visit http://creativecommons.org/licenses/by/4.0/. The Creative Commons Public Domain Dedication waiver (http://creativeco mmons.org/publicdomain/zero/1.0/) applies to the data made available in this article, unless otherwise stated in a credit line to the data. 


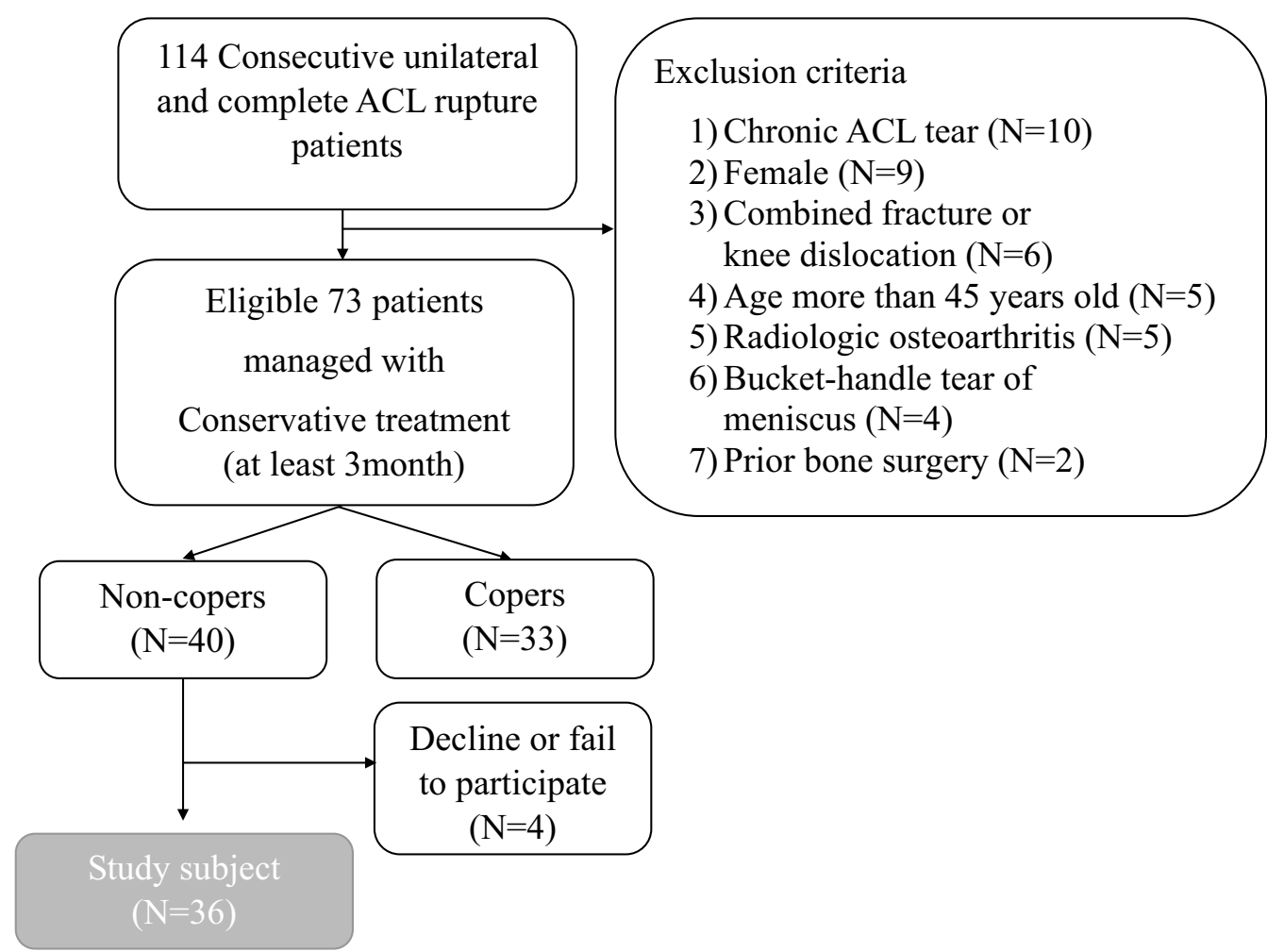

Fig. 1 Study design and eligibility criteria. Thirty-six patients were included in this cross-sectional study

meeting the following criteria were excluded: (1) chronic ACL tear (more than 6 months at first visit) $(N=10)$; (2) female sex (because of sex differences in gait properties [17-20]) $(N=9)$; (3) combined fracture or knee dislocation $(N=6)$; (4) aged more than 45 years $(N=5)$; (5) evidence of radiologic osteoarthritis (Kellegren-Lawrence grade II or more) $(N=5)$; (6) combined bucket-handle tear of meniscus $(N=4)$; and $(7)$ any prior surgery in the lower extremity $(N=2)$. After application of the exclusion criteria, 73 eligible patients remained. Initially, the patients were treated conservatively through physical therapy and strengthening exercise. Since the patients were transferred to our hospital after conservative treatment at another institution, the duration of conservative treatment varied in patients. After at least 3 months of conservative treatment, more than half of the patients ( 40 patients) complained of instability or giving-way, even though they had significantly reduced their activity level prior to injury. We defined these patients as "non-copers" in this paper and included them in the analysis [21]. The non-copers also underwent ACL reconstruction surgery after this study. Of the non-copers, four declined or failed to participate in the study. Therefore, 36 males with unilateral ACL rupture, who were functionally classified as non-copers, participated in this study. The mean subject age was 27.1 years ( \pm 7.0 , range $19-45$ years), the mean height was $172( \pm 7.2) \mathrm{cm}$, and the mean weight was 71.5 $( \pm 11.2) \mathrm{kg}$. The average range of motion (ROM) prior to gait laboratory analysis was $138.7^{\circ}\left( \pm 15.8^{\circ}\right)$. The average Lysholm score was $67.7( \pm 12.7)$. The pivot shift test results were Gr I for 11 patients, Gr II for 17 patients, and Gr III for 6 patients. The Lachmann test results were Gr I for 8 patients, Gr II for 22 patients, and Gr III for 6 patients.

\section{Data collection}

Gait data were measured in the Human Motion Analysis Lab at our institution. The average time interval between the injury and gait measurement was 4 months (range 3-8 months). Before gait measurements, subjects underwent conservative treatment until they met the following criteria: minimal knee effusion, no knee extension deficit, minimal pain in the injured limb with walking, and no visually identifiable gait impairments. The average pain score assessed on a numeric rating scale at the time of gait analysis was $1.2( \pm 0.8)$. Therefore, we could exclude any acute effects of knee trauma on the basis of the criteria above. Patients were asked to perform 5 min of easy walking to warm up. After warming up, reflective markers from a Helen Hayes marker set were placed on each 
subject's body [22]. Patients were asked to walk at their usual speed along a 9-m track.

In our gait analysis, we defined kinematic control as the different angulation of the knee joint and kinetic control as the change of moment by the action of the quadriceps. Motion (kinematic) data were acquired at a sample rate of $120 \mathrm{~Hz}$ using 12 charge-coupled device cameras equipped with a three-dimensional optical motion capture system (Motion Analysis, Santa Rosa, USA). Ground reaction force (kinetic) data were acquired at a sampling rate of $1200 \mathrm{~Hz}$ using three AMTI (Advanced Mechanical Technology Inc., Watertown, MA, USA) force plates. The kinetic data were then normalized by height and weight (\% body weight $\times$ height) [23].

We used Eva Real-Time software (Motion Analysis, Santa Rosa, USA), Microsoft Excel 2016 (Microsoft, Redmond, USA), and MATLAB R2017a (Mathworks, Natick, MA, USA) for real-time motion capture, post-processing, and marker data tracking. The average of three representative strides from five or six separate trials was used for the analysis of each session. Two kinematic gait parameters (knee flexion angle, knee rotation angle) and two kinetic parameters [internal knee extension moment (KEM), knee rotation moment] were measured.

\section{Statistical analysis}

Peak (maximum) kinematic and kinetic data of ACLruptured and uninjured limbs were compared using paired Student's $t$-tests. Four gait parameters, knee flexion angle, knee rotation angle, internal knee extension moment (KEM), and knee rotation moment were processed via principal component analysis (PCA). PCA was performed via two steps. First, features were extracted from each gait parameter. Second, each feature was scored for each subject. Each gait parameter consisted of an $n \times 101$ data matrix, where $n$ rows represent the number of cases and 101 columns represent standardized 101 timepoints. PCA processes this gait parameter matrix with an orthogonal transformation and extracts the data into a set of gait features that are linearly uncorrelated (principal components, PCs). These transformations allow the major features (PCs) of each gait parameter to be recognized. PCs were selected using a $90 \%$ trace criterion, and seven features were extracted from two gait parameters (i.e., KEM and knee flexion angle). The next step was the scoring of the extracted features. This was obtained by standardizing individual contributions to the extracted features [24]. In the case of a high score, the standardized individual contribution shows the same direction as the extracted feature. Conversely, when the score is low or negative, the contribution is applied the opposite direction. The PC score is a standardized score $($ mean $=0$, standard deviation $=1)$ that can be compared with other subjects or among the features. The standardized mean difference (SMD) of each of the PC scores was compared between the ACL-ruptured and uninjured knees. The feature that showed the highest SMD of the PC score was then investigated.

The PC scores of ACL-ruptured and uninjured limbs were compared using paired Student's $t$-tests. The normality of each PC score was assessed with the Kolmogorov-Smirnov test. All statistical analyses were performed using SPSS 19.0.1 for Windows (SPSS Inc., Chicago, IL, USA). $P$-values $<0.05$ were considered to indicate statistical significance.

\section{Results}

Notable kinematic patterns in terms of knee flexion angle and kinetic patterns of decreased KEM were observed during phases of the gait cycle. Analysis of the association between the kinematic and kinetic patterns at different gait phases revealed two distinctive kinematic strategies adopted by the ACL-injured knee.

Two distinctive kinematic patterns were observed in knee flexion angle (Table 1, Fig. 2b). Patients extended their ACL-ruptured knee more at the initial doublelimb stance (IDS) phase and then flexed more during the single-limb stance (SLS) to the terminal double-limb stance (TDS) phase (both $P<0.001$ ) compared with the contralateral uninjured knee (black and red arrows in Fig. 2b).

The most significant kinetic difference between the ACL-ruptured and uninjured knees was the peak-to-peak amplitude (i.e., difference between peak and trough) of KEM (Table 1, Fig. 2a). It was smaller in the ACL-ruptured knees, and the SMD was $1.02(P<0.001)$. The peak KEM in the ACL-ruptured knees was $27 \%$ smaller than that of the uninjured knees $(P<0.002$, ACL-ruptured: 2.5 (\%Body weight * Height), uninjured: 3.4 (\%Body weight * Height)).

We subsequently investigated the association between kinematic and kinetic parameters. At the IDS phase (i.e., loading phase), the peak knee flexion angles of the ACLruptured knees and uninjured knees were $17.1^{\circ}$ and $20.2^{\circ}$, respectively $(P<0.001)$. This angle was positively correlated with the peak KEM (Pearson $r=0.694, P<0.001$ ) (Fig. 3). In addition, knee flexion angle at SLS to TDS (i.e., KF PC3) was higher in ACL-ruptured knees $(P<0.001)$ and was negatively correlated with the KEM amplitude (i.e., KEM PC2) (Pearson $r=-0.710, P<0.001$ ) (Fig. 4).

Cumulatively, these data indicate that patients adopted two distinctive kinematic strategies to reduce KEM peak and amplitude of their ACL-ruptured knee. In the first strategy, patients extended their ACL-ruptured knee $3.1^{\circ}$ more at the IDS phase to reduce the peak KEM. In the second strategy, patients flexed their ACL-ruptured knee 
Table 1 Principal component analysis of kinetics and kinematics

\begin{tabular}{|c|c|c|c|c|c|}
\hline Gait measure & PC & Feature & $\begin{array}{l}\% \text { of variance } \\
\text { (cumulative) }\end{array}$ & $\begin{array}{l}\text { SMD } \\
(N=36)\end{array}$ & $P$-value* \\
\hline \multirow[t]{4}{*}{ (a) Knee extension moment (KEM) } & PC1 & Overall magnitude & 68.5 & 0.15 & 0.542 \\
\hline & PC2 & Amplitude of moment & 89.8 & 1.02 & $<0.001$ \\
\hline & PC3 & Phase shift & 95.6 & 0.11 & 0.483 \\
\hline & \multicolumn{5}{|c|}{ Interpretation: less amplitude of moment (of ACL ruptured limb) } \\
\hline \multirow[t]{4}{*}{ (b) Knee flexion angle } & PC1 & Overall flexion angle & 56.8 & 0.3 & 0.069 \\
\hline & PC2 & Flexion at swing phase & 75.9 & 0.26 & 0.157 \\
\hline & PC3 & Flexion at SLS to TDS phase & 88.2 & 0.87 & $<0.001$ \\
\hline & PC4 & Flexion at IDS phase & 94.8 & 0.76 & $<0.001$ \\
\hline
\end{tabular}

$P C$ principal component, SMD standardized mean difference, IDS initial double-limb support, SLS single-limb support, TDS terminal double-limb support, IR internal rotation

${ }^{*}$ Comparisons were made with paired $t$-test. Bold face indicates statistical significance
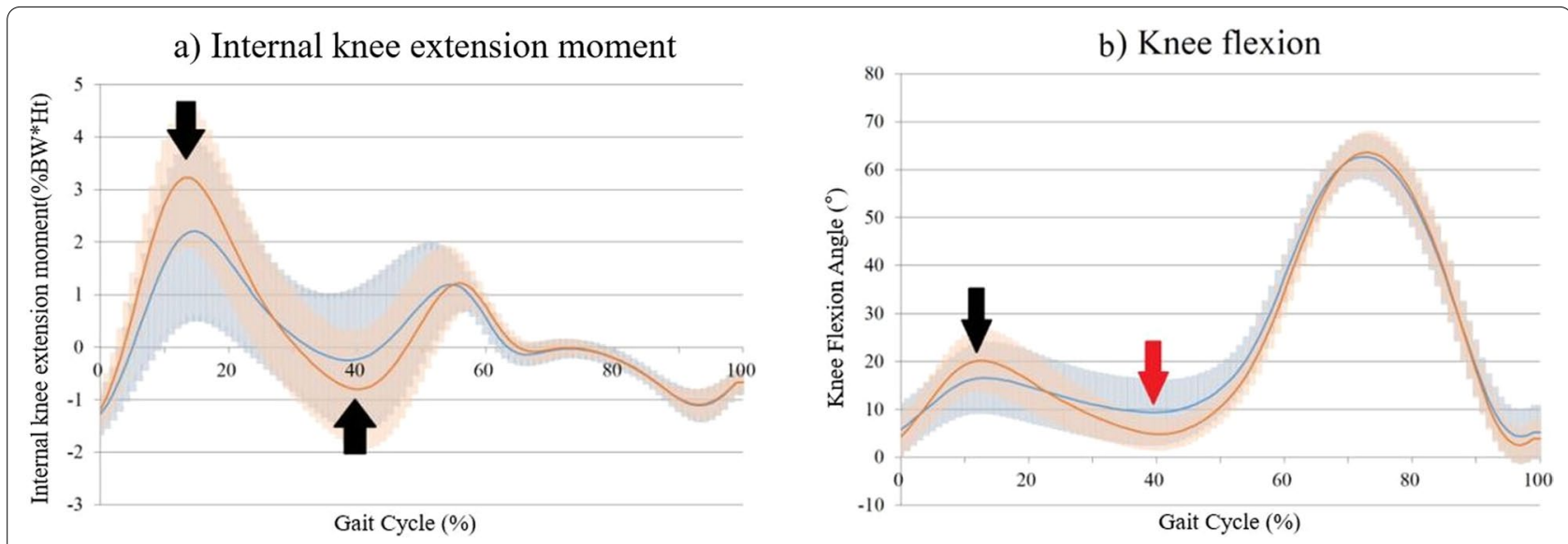

Fig. 2 Kinetics and kinematics of the knee joint in the sagittal and axial planes. The blue curve indicates the ACL-ruptured limb, and the red curve indicates the contralateral uninjured limb. The shaded region represents mean \pm one standard deviation. Table 1 presents a statistical analysis of the graph. a Knee extension moment. The knee extension moment peak value and amplitude were both smaller in the ACL-ruptured limb (black arrow). b Knee flexion angle. The ACL-ruptured knee showed extension at the IDS phase (black arrow) and more flexion from the SLS to the TDS phase (red arrow)

at SLS to TDS phase to reduce the KEM amplitude. Linear regression analysis showed that the adjusted $R^{2}$ of the first strategy was 0.475 and that of the second strategy was 0.497 (Table 2).

\section{Discussion}

The most important findings of this study are that we found two distinct kinematic deviations on ACL-ruptured knee associated with the peak and amplitude of KEM, respectively. At IDS phase of the gait cycle, kinematic strategy to extend the knee contributed to the reduction of peak KEM, showing consistency with the quadriceps avoidance strategy. At SLS to TDS phase, kinematic strategy to flex the knee leads to reduced KEM amplitude, reflecting the stiffening strategy. Our findings suggest that kinematic control of knee joint is an important gait deviation mechanism in patients with ACL rupture.

. To our knowledge, this is the first study to describe two distinctive kinematic controls associated with kinetics in patients with ACL rupture. Berchuck et al. found that the KEM was decreased and sometimes even reversed in the mid-stance walking phase of patients with ACL rupture, terming this phenomenon "quadriceps avoidance gait" [6]. However, subsequent studies did not yield consistent results. Studies by Georgoulis et al. (and others) found no difference in sagittal-plane kinematics, while studies by Hurd et al. (and others) found 

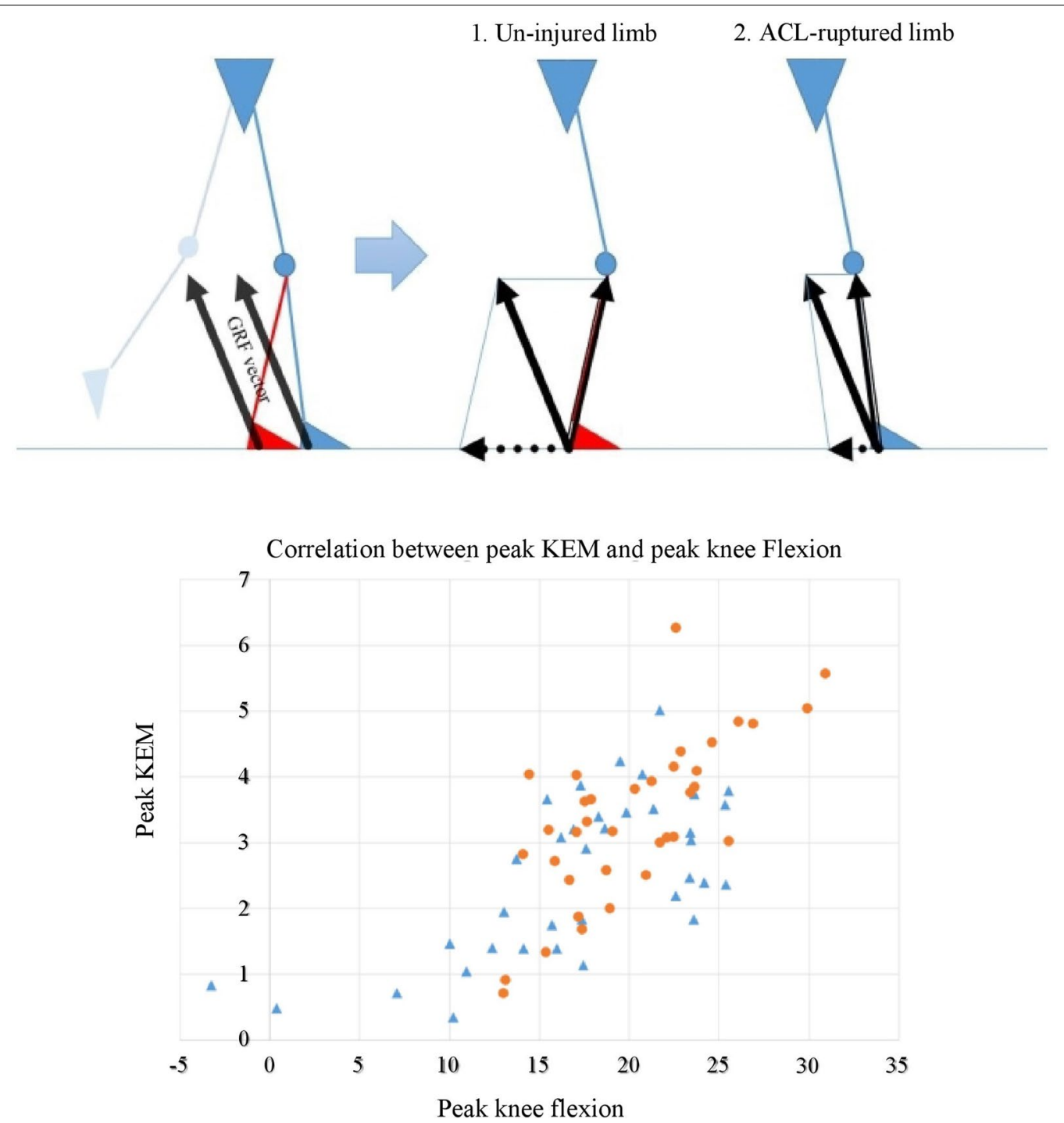

Fig. 3 Schematic representations of knee extension moment (KEM) and knee flexion angle at the initial double-limb stance (IDS) phase. During this phase, ground reaction force (GRF) is generated for the repulsive body weight force (black arrow). The GRF can be divided into an axial vector (double arrow) and a transverse vector (dotted arrow). The axial vector runs parallel to the tibia and acts as a compressive force to the tibiofemoral joint. The transverse vector runs parallel to the ground and acts as a knee flexion force (counter to the knee extension moment by the quadriceps) The ACL-ruptured knee can be unstable during this phase, so patients try to reduce the transverse vector by extending their knee (note the difference in knee flexion angle). Instead, the tibiofemoral joint axial force can be increased. The graph shows the correlations between peak KEM and peak knee flexion at the IDS phase. The blue triangle represents the ACL-ruptured limb, and the orange circle represents the uninjured limb. Note the strong correlation between the two variables (Pearson $r=0.694, P<0.001$ ). Linear regression analysis showed that the adjusted $R^{2}$ value of the first strategy was 0.475

significant KEM reduction [3-13]. More recent reports with delicate study designs found that patients with ACL rupture had a reduced KEM, although the KEM was still greater than zero and was not reversed, as in the original article $[5,8,13]$. We noticed that the studies that did not observe the reduction of KEM used different patient selection criteria and/or different gait examination timing. For example, Georgoulis and colleagues performed the gait examinations on average at $7.6 \pm 4.3$ weeks after ACL rupture and did not classify patients as copers or non-copers [12]. Furthermore, Berchuck et al. found normal biphasic patterns in $25 \%$ of the analyzed patients, suggesting that pattern results may vary according to patient selection criteria [6]. In the present work, we excluded females, copers, and acute or chronic ACL rupture to minimize these possible confounding effects. 


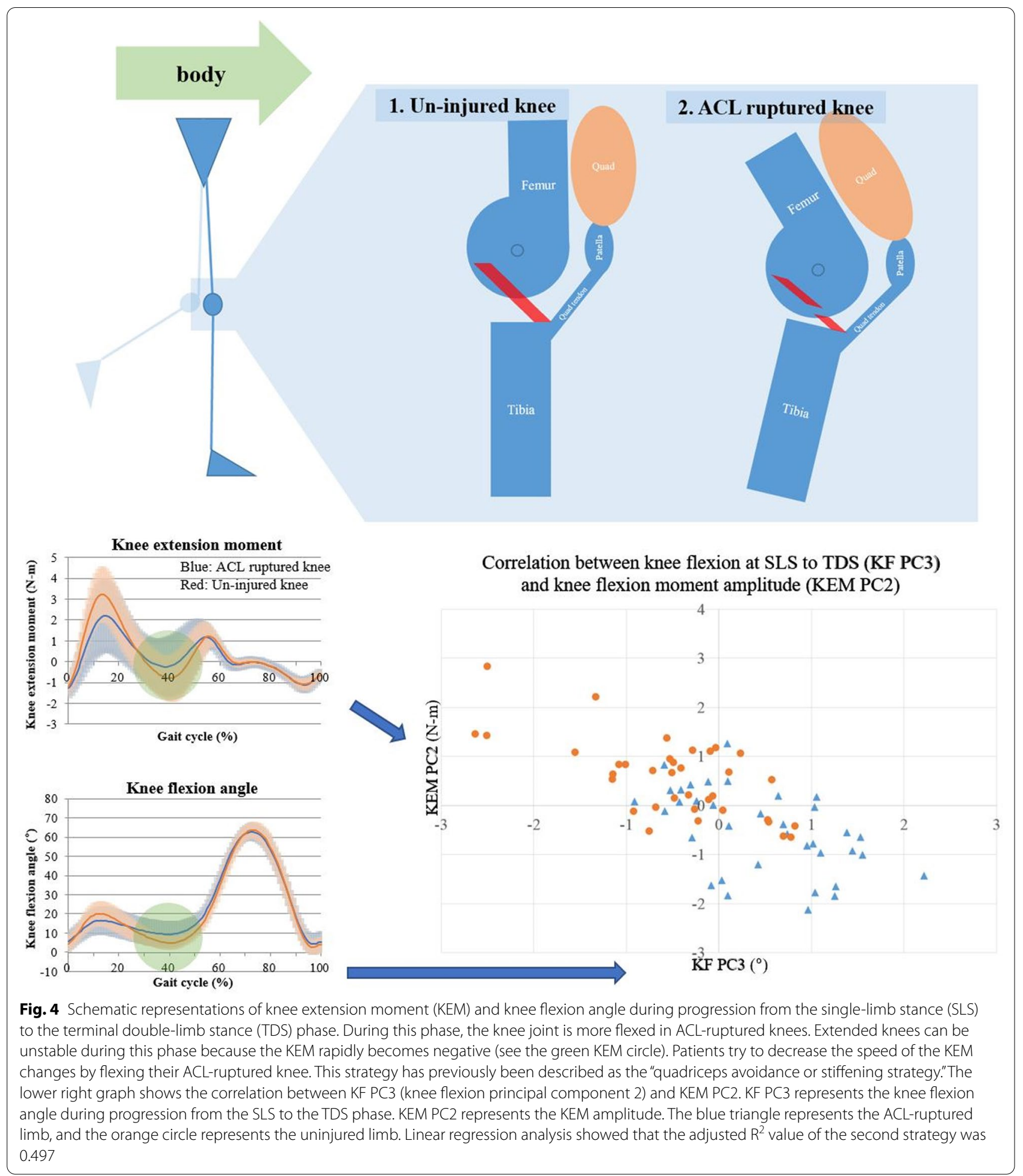

As described above, gait features differ according to sex [17-20]. An acute ACL rupture can result in antalgic gait, whereas chronic ACL rupture can result in arthritic gait features [3, 12]. Proper control and selection of the patient group are very important. Further studies analyzed the kinetic patterns of quadriceps avoidance gait by examining muscle strength and electromyography (EMG) $[5,16]$. However, kinematic control has not been widely studied, even though this type of control is one 
Table 2 Regression model for kinematic strategy

\begin{tabular}{|c|c|c|c|}
\hline \multirow[t]{2}{*}{ Regression models } & \multicolumn{3}{|l|}{ Peak KEM } \\
\hline & $B \pm S E^{*}$ & $P$-value & $R^{2} \mathrm{adj} \dagger$ \\
\hline \multirow[t]{3}{*}{ (1) Knee flexion at IDS } & $0.152 \pm 0.019$ & $<0.001$ & 0.475 \\
\hline & \multicolumn{3}{|c|}{ Amplitude of KEM } \\
\hline & $B \pm S E^{*}$ & $P$-value & $R^{2}$ adj† \\
\hline (2) Knee flexion at SLS to TDS & $-0.731 \pm 0.087$ & $<0.001$ & 0.497 \\
\hline
\end{tabular}

"Values are given as the $B$ (standardized regression coefficient) and SE (standard error)

${ }^{\dagger} R^{2}$ adj $=\%$ variance explained by each variable

of the main means of neuromuscular control in patients with ACL rupture.

To understand the gait deviation of patients with ACL rupture, one must examine the mechanism involved when the internal KEM decreases. The most common interpretation is direct inhibition of the quadriceps femoris $[5,16]$. The internal KEM is generated by eccentric contraction of the quadriceps with a moment opposite to the external knee flexion moment (KFM), which acts as an external flexion force in the loading phase. The KEM has the same size as the KFM, but the opposite mechanical balance. During gait, the KEM can act as an anterior translation force for the proximal tibia; thus, the quadriceps is unconsciously suppressed in the patients with ACL rupture $[5,6,16]$. In support of this hypothesis, studies using EMG have shown that quadriceps muscle activity is suppressed in patients with ACL rupture [15, 16]. In addition, increased hamstring activity is associated with this suppression. This increase in muscle activity is referred to as muscle coactivation; both phenomena are considered major neuromuscular adaptations in patients with ACL rupture [5, 14-16, 25].

Unlike the study by Berchuck et al. that described the quadriceps avoidance pattern at the mid-stance phase, our study, as well as previous studies, reported knee extensions at the IDS phase $[8,10,14,15]$. We investigated the relationship between peak KEM and peak knee flexion angle in the IDS phase and found a strong linear relationship (Pearson $r=0.694, P<0.001$ ). The results showed that knee extension in the IDS phase reduces the KEM. Extension of the knee in the IDS phase has been observed in previous studies but was not previously interpreted mathematically as in the present study $[3,5,10]$. These results suggest that both kinematic control and kinetic control may be associated with the gait of patients with ACL rupture. In the uninjured leg, knee flexion occurs with quadriceps eccentric contraction to reduce GRF during the IDS phase. However, an ACLruptured leg creates knee extension at the IDS phase to minimize the use of the quadriceps, and consequently reduces KEM. When the knee is further extended, the transverse vector decreases, reducing the force applied to the anteroposterior direction of the tibia [6] (Fig. 3). However, this reduction is expected to increase the GRF distribution in the axial direction (while decreasing the transverse vector). This increased GRF distribution may increase the impact on the tibiofemoral (TF) joint and may also contribute to the development of TF arthritis or subsequent meniscus injury after ACL rupture $[10,11]$. In conclusion, this study is meaningful in interpreting the quadriceps avoidance gait at the IDS phase from the viewpoint of kinematic control. This phenomenon likely corresponds to a centrally controlled mechanism of the knee joint [26]. A study in a rat model showed that the central nervous system (CNS) regulates muscle activation to reduce the load within the joint [27]. We think that similar regulation occurs centrally in ACL-injured knees in terms of kinematic control of the joint. This strategy could be a coordinated way to reduce peak KEM in the early stages through feed-forward signaling at the IDS phase by kinematic control.

After the IDS phase, the walking strategy from the SLS to the TDS phase was similar to the stiffening strategy described by Hurd et al $[5,6,8,15]$. However, the knee stiffening strategy in the SLS to TDS phases seems to affect the amplitude rather than the KEM peak value. When the two walking strategies were modeled by regression analysis (Table 2 ), the adjusted $R^{2}$ values were 0.475 and 0.497 . These correlations could account for significant portions of the KEM peak and amplitude. The rest of the KEM peak and amplitude are likely to be due to direct inhibition or muscle coactivation, which were not included in this study. The correlation has been observed by others [5].

This study has some limitations. First, participants were restricted to non-coping men. This selection limits the degree to which the results of this study can be applied to other groups. Women are known to have 
greater rotational laxity than men; therefore, the results may be different in women [17-20]. However, since men and women have different gait patterns and skeletal alignments, analyzing men and women together without controls could make the results harder to interpret [17-20]. Future research should focus on women. Second, only non-copers were tested. Nonetheless, analysis of the gait pattern of copers is not as important as that of non-copers at present. Moreover, analysis that fails to discriminate non-copers from copers could lead to inadequate conclusions. Third, the gait and clinical tests were performed between 3 and 8 months after the injury, meaning that pain and stiffness may have affected the gait. As a retrospective study, the patients arrived at the institution at different timepoints following the injury. The result was that the conservative treatment at other hospitals prior to the first visit varied. However, before gait measurements, each participant was verified to have minimal knee effusion, no knee extension deficits, minimal pain in the injured limb with walking, and no visually identifiable gait impairments. These criteria were applied to minimize the effects of pain and stiffness. The average pain numeric rating was $1.2 \pm 0.8$, and the average range of motion (ROM) prior to gait analysis in the laboratory was $138.7 \pm 15.8^{\circ}$. However, the gait patterns of patients with acute or chronic ACL ligament rupture may be different; therefore, further studies are needed [3, 12]. This study does not have healthy subjects as a control; instead, contralateral uninjured limb was used. ACL rupture can affect the gait pattern of the opposite limb as well. Having a control group of healthy individuals will enhance our understanding of the gait deviation in patients with ACL injury. In the future, we hope to expand the studies with healthy subjects included. Lastly, quadriceps atrophy may be present in patients with ACL injury. No subject had severe observable quadriceps atrophy, especially because the time between the injury and the gait study was short. Nevertheless, quantitative assessment of quadriceps atrophy would allow the assessment of possible confounding effects that the atrophy may have on the gait analysis.

\section{Conclusions}

Patients showed two distinct kinematic deviations to reduce the KEM peak and amplitude of their ACLruptured knee. This suggests that kinematic control of knee joint is an important gait deviation mechanism of patients with ACL rupture. Our findings should be taken into consideration when treating patients with ACL ruptured conservatively.

\section{Abbreviations}

ACL: Anterior cruciate ligament; EMG: Electromyography; GRF: Ground reaction force; IDS: Initial double limb stance; KEM: Knee extension moment; KFM: Knee flexion moment; MRI: Magnetic resonance imaging; PC: Principal component; PCA: Principal component analysis; ROM: Range of motion; SLS: Single-limb stance; SMD: Standardized mean deviation; TDS: Terminal doublelimb stance; TF: Tibiofemoral.

\section{Acknowledgements}

We thank Seong Hyun Kim, Hye Sun Park, and Hyo Jeong Yoo for their technical support to collect and analyze the kinematic data from the participants. We appreciate statistical consultation provided by the Medical Research Collaborating Center at the Seoul National University College of Medicine. We are also grateful to Eun Soo Ahn for help in proofreading and correcting the manuscripts.

\section{Authors' contributions}

JHP was a major contributor in writing and revision of the manuscript. MC acquired and interpreted the data. JL and $\mathrm{HH}$ performed the statistical analysis. ML designed the study and supervised. DHR designed the study and contributed in writing - review and editing. All authors read and approved the final manuscript.

\section{Funding}

None.

Availability of data and materials

The datasets used and/or analyzed during the current study are available from the corresponding author on reasonable request.

\section{Declarations}

\section{Ethics approval and consent to participate}

This study was approved by the Seoul National University College of Medicine/Seoul National University Hospital Institutional Review Board (IRB no. H-1706-177-864). Each participant gave written, informed consent to participate.

\section{Consent for publication}

Not applicable.

\section{Competing interests}

The authors declare that they have no competing interests.

\section{Author details}

${ }^{1}$ Department of Orthopaedic Surgery, Seoul National University Hospital, 101 Daehak-ro, Jongno-gu, Seoul 110-744, Korea. ${ }^{2}$ Seoul National University College of Medicine, Seoul, South Korea.

Received: 16 April 2021 Accepted: 8 December 2021

Published online: 24 December 2021

\section{References}

1. Leblanc MC, Kowalczuk M, Andruszkiewicz N et al (2015) Diagnostic accuracy of physical examination for anterior knee instability: a systematic review. Knee Surg Sports Traumatol Arthrosc 23:2805-2813

2. Kuroda R, Hoshino Y, Kubo S et al (2012) Similarities and differences of diagnostic manual tests for anterior cruciate ligament insufficiency: a global survey and kinematics assessment. Am J Sports Med 40:91-99

3. Ismail SA, Button K, Simic M et al (2016) Three-dimensional kinematic and kinetic gait deviations in individuals with chronic anterior cruciate ligament deficient knee: a systematic review and meta-analysis. Clin Biomech 35:68-80

4. Hart HF, Culvenor AG, Collins NJ et al (2016) Knee kinematics and joint moments during gait following anterior cruciate ligament reconstruction: a systematic review and meta-analysis. Br J Sports Med 50:597-612 
5. Hurd WJ, Snyder-Mackler L (2007) Knee instability after acute ACL rupture affects movement patterns during the mid-stance phase of gait. J Orthop Res 25:1369-1377

6. Berchuck M, Andriacchi TP, Bach BR et al (1990) Gait adaptations by patients who have a deficient anterior cruciate ligament. J Bone Jt Surg Am 72:871-877

7. Ferber R, Osternig LR, Woollacott MH et al (2002) Gait mechanics in chronic ACL deficiency and subsequent repair. Clin Biomech 17:274-285

8. Hart JM, Ko JW, Konold T et al (2010) Sagittal plane knee joint moments following anterior cruciate ligament injury and reconstruction: a systematic review. Clin Biomech 25:277-283

9. Torry MR, Decker MJ, Ellis HB et al (2004) Mechanisms of compensating for anterior cruciate ligament deficiency during gait. Med Sci Sports Exerc 36:1403-1412

10. Noehren B, Wilson H, Miller C et al (2013) Long-term gait deviations in anterior cruciate ligament-reconstructed females. Med Sci Sports Exerc 45:1340-1347

11. Podraza JT, White SC (2010) Effect of knee flexion angle on ground reaction forces, knee moments and muscle co-contraction during an impactlike deceleration landing: implications for the non-contact mechanism of ACL injury. Knee 17:291-295

12. Georgoulis AD, Papadonikolakis A, Papageorgiou CD et al (2003) Threedimensional tibiofemoral kinematics of the anterior cruciate ligamentdeficient and reconstructed knee during walking. Am J Sports Med 31:75-79

13. Roewer BD, Di Stasi SL, Snyder-Mackler L (2011) Quadriceps strength and weight acceptance strategies continue to improve two years after anterior cruciate ligament reconstruction. J Biomech 44:1948-1953

14. Rudolph KS, Axe MJ, Buchanan TS et al (2001) Dynamic stability in the anterior cruciate ligament deficient knee. Knee Surg Sports Traumatol Arthrosc 9:62-71

15. Gardinier ES, Manal K, Buchanan TS et al (2012) Gait and neuromuscular asymmetries after acute anterior cruciate ligament rupture. Med Sci Sports Exerc 44:1490-1496

16. Shanbehzadeh S, Mohseni Bandpei MA, Ehsani F (2017) Knee muscle activity during gait in patients with anterior cruciate ligament injury: a systematic review of electromyographic studies. Knee Surg Sports Traumatol Arthrosc 25:1432-1442

17. Hsu WH, Fisk JA, Yamamoto Y et al (2006) Differences in torsional joint stiffness of the knee between genders: a human cadaveric study. Am J Sports Med 34:765-770

18. Ro DH, Lee DY, Moon G et al (2017) Sex differences in knee joint loading: cross-sectional study in geriatric population. J Orthop Res 35:1283-1289

19. Park HS, Wilson NA, Zhang LQ (2008) Gender differences in passive knee biomechanical properties in tibial rotation. J Orthop Res 26:937-944

20. Shultz SJ, Shimokochi Y, Nguyen AD et al (2007) Measurement of varusvalgus and internal-external rotational knee laxities in vivo - part II: relationship with anterior-posterior and general joint laxity in males and females. J Orthop Res 25:989-996

21. Kaplan Y (2011) Identifying individuals with an anterior cruciate ligamentdeficient knee as copers and noncopers: a narrative literature review. J Orthop Sports Phys Ther 41:758-766

22. Kadaba MP, Ramakrishnan HK, Wootten ME (1990) Measurement of lower extremity kinematics during level walking. J Orthop Res 8:383-392

23. Wannop JW, Worobets JT, Stefanyshyn DJ (2012) Normalization of ground reaction forces, joint moments, and free moments in human locomotion. J Appl Biomech 28:665-676

24. Deluzio KJ, Astephen JL (2007) Biomechanical features of gait waveform data associated with knee osteoarthritis: an application of principal component analysis. Gait Posture 25:86-93

25. Boerboom AL, Hof AL, Halbertsma JP et al (2001) Atypical hamstrings electromyographic activity as a compensatory mechanism in anterior cruciate ligament deficiency. Knee Surg Sports Traumatol Arthrosc 9:211-216

26. Frey-Law LA, Avin KG (2013) Muscle coactivation: a generalized or localized motor control strategy? Muscle Nerve 48:578-585

27. Kalytczak MM, Lucareli PR (2016) Kinematic and electromyographic analysis in patients with patellofemoral pain syndrome during single leg triple hop test. Gait Posture 49:246-251

\section{Publisher's Note}

Springer Nature remains neutral with regard to jurisdictional claims in published maps and institutional affiliations.
Ready to submit your research? Choose BMC and benefit from:

- fast, convenient online submission

- thorough peer review by experienced researchers in your field

- rapid publication on acceptance

- support for research data, including large and complex data types

- gold Open Access which fosters wider collaboration and increased citations

- maximum visibility for your research: over $100 \mathrm{M}$ website views per year

At BMC, research is always in progress.

Learn more biomedcentral.com/submissions 